\title{
A multi-phase field model of planar dislocation networks
}

\author{
M Koslowski ${ }^{1}$ and M Ortiz \\ Division of Engineering and Applied Science, California Institute of Technology, Pasadena, \\ CA 91125, USA
}

Received 20 January 2004

Published 31 August 2004

Online at stacks.iop.org/MSMSE/12/1087

doi:10.1088/0965-0393/12/6/003

\begin{abstract}
In this paper we extend the phase-field model of crystallographic slip of Ortiz (1999 J. Appl. Mech. ASME 66 289-98) and Koslowski et al (2001 J. Mech. Phys. Solids 50 2957-635) to slip processes that require the activation of multiple slip systems, and we apply the resulting model to the investigation of finite twist boundary arrays. The distribution of slip over a slip plane is described by means of multiple integer-valued phase fields. We show how all the terms in the total energy of the crystal, including the long-range elastic energy and the Peierls interplanar energy, can be written explicitly in terms of the multi-phase field. The model is used to ascertain stable dislocation structures arising in an array of finite twist boundaries. These structures are found to consist of regular square or hexagonal dislocation networks separated by complex dislocation pile-ups over the intervening transition layers.
\end{abstract}

(Some figures in this article are in colour only in the electronic version)

\section{Introduction}

The scope of the work presented in this paper is twofold. First, we extend the phase-field model of crystallographic slip of Ortiz [1] and Koslowski et al [2] to slip processes that require the activation of multiple slip systems. Second, we apply the resulting model to the investigation of twist boundaries of finite extent. Ortiz [1], and later Koslowski et al [2], proposed the use of an integer-valued phase field as a device for describing complex dislocation arrangements on a slip plane. In this representation, the value of the phase field at a point on the slip plane counts the number of dislocations that have crossed over the point, accounting for the sign of the crossing. From the standpoint of kinematics of plastic deformation, the phase field may variously be regarded as a normalized displacement jump across the slip plane in the direction of the Burgers vector or as a normalized slip strain concentrated on the slip plane. Finally, the present notion of a phase field emanates directly from the Peierls concept of an interplanar potential as the energetic basis for the formation of stable discrete dislocations

1 Present address: Theoretical Division, Los Alamos National Laboratory, Los Alamos, NM 87545, USA. 
in crystals. Owing to the periodicity of the lattice, the constrained Peierls potential may be expected to itself be periodic and hence possess a countable number of minima, or wells. Each well corresponds to slip by an integer multiple of the Burgers vector. Within this energetic framework, the value of the phase field at a point designates the well, or phase, the point is in, hence the term phase field.

When more than one slip system becomes active on a slip plane, the description of crystallographic slip requires the use of multiple phase fields, each representing the activity on one of the slip systems. This multi-phase field representation provides the precise information required to evaluate all the terms in the total energy of the crystal: the long-range elastic energy; the Peierls interplanar energy; and the interaction energy with an applied or built-in elastic stress field.

Dislocations in ductile single crystals are often observed to arrange themselves into lowenergy configurations in the form of planar networks and sub-grain boundaries [3-5]. Energy minimization provides a means of understanding these relaxed dislocation structures $[6,7]$. Inelastic effects such as hysteresis and the Bauschinger effect can also be investigated within a similar variational framework through the interaction of a dislocation ensemble and randomly distributed obstacles [2,8].

Dislocation networks can play an important role in the effective response of crystals $[9,10]$. For instance, Bulatov and Cai [9] have shown that co-planar dislocations can increase their mobility by reacting to form networks. From atomistic simulations they have found that the critical stress to move a network in a [110]-plane of bcc molybdenum is $50 \%$ smaller than that required to move an isolated dislocation.

In a continuum setting, sub-grain dislocation networks have been studied analytically $[4,5,11-13]$ and computationally [9, 14-16]. Analytical studies have been for the most part limited to infinite planar dislocation arrays and based on simple kinematics and geometrical arguments such as Frank's relation or on line-tension models that neglect elastic interactions and the structure of the dislocation nodes $[4,11,13]$. Computational models that regard dislocations as elastic line defects are often beset by the cost of evaluating the long-range elastic interactions between each pair of dislocation segments and by the sensitivity of the results to the choice of the interaction cut-off radius [14].

By way of illustration, we apply the multi-phase field model to the investigation twist boundaries. The specific geometry under consideration in this work consists of a periodic array of twist boundaries of finite size, each of which is forced to undergo a prescribed twist angle. The periodic cells may be regarded as loosely representing individual grain boundaries in a polycrystalline material. While this geometry is somewhat contrived and is chosen mainly for computational convenience, it nevertheless gives rise to a nontrivial dislocation pattern and it serves to illustrate the range and scope of this model. Of primary interest in this problem is whether regular dislocation networks are established within the twist regions, the size and geometry of the networks, and the geometry of the transition pile-ups that form at cell boundaries.

The operative principle determinant of the relaxed dislocation arrangements is assumed to be energy minimization. The dislocation pattern is therefore governed by the following: the long-range elastic interaction between dislocation segments; the structure and energy of dislocation nodes; and the angle of misorientation between the crystals. The stable dislocation configurations are computed by means of a Metropolis Monte Carlo algorithm. We consider slip system geometries that result in square and hexagonal dislocation networks [3-5]. The ability of the approach to predict complex dislocation patterns is noteworthy. A dimensional analysis of the energy also reveals useful scaling relations involving the cell size, Burgers vector size, and twist angle. 


\section{Multi-phase field representation of crystallographic slip}

In this section we introduce the multi-phase field representation of crystallographic slip and attendant dislocation structures. As in the previous work of Ortiz [1] and Koslowski et al [2], we consider slip processes occurring on a single slip plane. However, we extend the work of Koslowski et al [2] to allow for simultaneous activity on more than one slip system through the use of multiple phase fields. The primary objective of this section is to obtain an explicit expression for the total energy of the crystal in terms of the multi-phase field.

In the linearized kinematics approximation, the plastic deformation in a crystal admits the representation

$$
\beta_{i j}^{p}=\sum_{\alpha=1}^{n} \gamma^{\alpha} s_{i}^{\alpha} m_{j}^{\alpha},
$$

where $\gamma^{\alpha}, \boldsymbol{s}^{\alpha}$, and $\boldsymbol{m}^{\alpha}$ are the slip strains, slip direction, and slip-plane normal corresponding to slip system $\alpha$, and $n$ is the number of slip systems of the crystal. The slip directions are in turn defined as $\boldsymbol{s}^{\alpha}=\boldsymbol{b}^{\alpha} / b^{\alpha}$, where $\boldsymbol{b}^{\alpha}$ is the Burgers vector corresponding to slip system $\alpha$ and $b^{\alpha}=\left|\boldsymbol{b}^{\alpha}\right|$. Nye's dislocation density tensor [17] follows from the plastic deformation as

$$
\alpha_{i l}=\beta_{i j, k}^{p} e_{k j l} \text {, }
$$

where, here and subsequently, a comma denotes partial differentiation and $e_{k j l}$ are the components of the permutation tensor. The defining property of $\boldsymbol{\alpha}$ is that

$$
b_{i}(\Sigma)=\int_{\Sigma} \alpha_{i l} n_{l} \mathrm{~d} S
$$

where $\boldsymbol{b}(\Sigma)$ is the total Burgers vector of the dislocations crossing the oriented surface $\Sigma$.

The elastic energy of the plastically deformed crystal is

$$
E=\int \frac{1}{2} c_{i j k l}\left(u_{i, j}-\beta_{i j}^{p}\right)\left(u_{k, l}-\beta_{k l}^{p}\right) \mathrm{d}^{3} x,
$$

where $\boldsymbol{u}$ is the displacement field, $c_{i j k l}=c_{j i k l}=c_{i j l k}=c_{k l i j}$ are the elastic moduli of the crystal, and the crystal is assumed to be of infinite extent. Minimizing out the displacement field with the aid of the Fourier transform gives the reduced energy [2]

$$
E^{\mathrm{int}}=\frac{1}{(2 \pi)^{3}} \int \frac{1}{2} \hat{A}_{m n u v}(\boldsymbol{k}) \hat{\beta}_{m n}^{p}(\boldsymbol{k}) \hat{\beta}_{u v}^{p *}(\boldsymbol{k}) \mathrm{d}^{3} k,
$$

where, here and subsequently, a superposed ( $)$ denotes the Fourier transform of a function and

$$
\hat{A}_{\text {mnuv }}(\boldsymbol{k})=c_{\text {mnuv }}-c_{k l u v} c_{i j m n} \hat{G}_{k i}(\boldsymbol{k}) k_{j} k_{l} .
$$

In this expression, $\boldsymbol{G}(\boldsymbol{x})$ is the Green's tensor of linear elasticity, and $\hat{\boldsymbol{G}}(\boldsymbol{k})$ its Fourier transform. Mura [18] has shown that $E^{\text {int }}$ can also be expressed in terms of Nye's dislocation density tensor (2.2).

Suppose now that the plastic deformation of the crystal is confined to a single plane, which, for definiteness, we take to be the $\left(x_{1}, x_{2}\right)$-plane. Then, the plastic deformation tensor is of the form

$$
\beta_{i j}^{p}(\boldsymbol{x})=\sum_{\alpha=1}^{n} \gamma^{\alpha}\left(x_{1}, x_{2}\right) s_{i}^{\alpha} \delta_{3 j} \delta_{\mathrm{D}}\left(x_{3}\right),
$$

where $\delta_{i j}$ are the components of Kronecker's delta, and $\delta_{\mathrm{D}}$ denotes Dirac's delta distribution. We additionally introduce the representation

$$
\gamma^{\alpha}=\frac{b^{\alpha}}{d^{\alpha}} \zeta^{\alpha},
$$


where $d^{\alpha}$ is the interplanar distance across the slip plane and $\zeta^{\alpha}$ may be regarded as a normalized slip distribution function. Using this representation equation (2.7) becomes

$$
\beta_{i j}^{p}(\boldsymbol{x})=\sum_{\alpha=1}^{n} \frac{1}{d} \zeta^{\alpha}\left(x_{1}, x_{2}\right) b_{i}^{\alpha} \delta_{3 j} \delta_{\mathrm{D}}\left(x_{3}\right) .
$$

Integrating the displacement gradients across the slip plane and taking into account (2.9) and the boundedness of the elastic deformations, we further obtain the identity

$$
\llbracket u_{i} \rrbracket\left(x_{1}, x_{2}\right)=\sum_{\alpha=1}^{n} \zeta^{\alpha}\left(x_{1}, x_{2}\right) b_{i}^{\alpha},
$$

which gives the displacement jump across the slip plane in terms of the slip fields. Inserting (2.1) into (2.5), the elastic energy specializes to

$$
E^{\mathrm{int}}[\boldsymbol{\zeta}]=\frac{1}{(2 \pi)^{2}} \int \frac{1}{2} \hat{\boldsymbol{\zeta}}^{\mathrm{T}} \boldsymbol{A} \hat{\boldsymbol{\zeta}}^{*} \mathrm{~d}^{2} k,
$$

where

$$
A_{\alpha \beta}\left(k_{1}, k_{2}\right)=\int_{-\infty}^{\infty} \hat{A}_{i 3 k 3}(\boldsymbol{k}) b_{i}^{\alpha} b_{k}^{\beta} \mathrm{d} k_{3}
$$

are interaction coefficients, and, for convenience, we collect all normalized slip functions into the multi-slip field $\zeta \equiv\left\{\zeta^{1}, \ldots, \zeta^{n}\right\}$.

Following [2], we suppose that, in addition to the elastic energy, the short-range interatomic interactions across the slip plane introduce an additional term in the energy that can be approximated by the piece-wise quadratic Peierls potential [19]

$$
\phi(\boldsymbol{\zeta}, \boldsymbol{\xi})=\sum_{\alpha=1}^{n} \min _{\xi^{\alpha} \in \mathbb{Z}} \frac{1}{2} C^{\alpha}\left(\zeta^{\alpha}-\xi^{\alpha}\right)^{2}
$$

where

$$
C^{\alpha}=\frac{1}{d^{\alpha}} c_{i 3 k 3} b_{i}^{\alpha} b_{k}^{\alpha}
$$

and $\xi^{\alpha}$ is an integer-valued phase field for slip system $\alpha$. The Peierls potential (2.13) is a periodic function in each of the variables $\zeta^{\alpha}$ with a countable number of minima, or wells. The function $\xi^{\alpha}\left(x_{1}, x_{2}\right)$ labels the well, or phase, of the $\alpha$ system that the point $\left(x_{1}, x_{2}\right)$ is in, hence the term 'phase field'. The interplanar energy has the effect of forcing the slip fields $\zeta^{\alpha}$ to take near-integer values close to $\xi^{\alpha}$. Thus, the corresponding displacement jump (2.10) across the slip plane consists of near-integer multiples of the Burgers vectors separated by rapid transitions, or cores, across the dislocation lines. The field $\xi^{\alpha}\left(x_{1}, x_{2}\right)$ may also be regarded as a counter that keeps track of the number of dislocations of type $\alpha$ that have crossed over the point $\left(x_{1}, x_{2}\right)$. For notational convenience, we introduce the diagonal matrix

$$
C_{\alpha \beta}= \begin{cases}C^{\alpha}, & \text { if } \beta=\alpha, \\ 0, & \text { otherwise, }\end{cases}
$$

whereupon the Peierls potential takes the form

$$
\phi(\boldsymbol{\zeta}, \boldsymbol{\xi})=\min _{\boldsymbol{\xi} \in \mathbb{Z}^{n}} \frac{1}{2}(\boldsymbol{\zeta}-\boldsymbol{\xi})^{\mathrm{T}} \boldsymbol{C}(\boldsymbol{\zeta}-\boldsymbol{\xi}) .
$$

The total interplanar energy is

$$
E^{\mathrm{core}}[\boldsymbol{\zeta}, \boldsymbol{\xi}]=\int \phi(\boldsymbol{\zeta}, \boldsymbol{\xi}) \mathrm{d}^{2} x,
$$

which measures the misfit or core energy of the dislocations. 
In the absence of applied stresses, the total energy of the crystal is the sum of its elastic interaction energy and its interplanar energy, i.e.

$$
E=E^{\text {core }}+E^{\text {int }} \text {. }
$$

Inserting (2.11) and (2.17) in (2.18) leads to the energy

$$
E[\zeta]=\inf _{\xi \in X} E[\zeta \mid \xi],
$$

where $X$ represents some suitable configuration space of integer-valued phase fields and

$$
E[\boldsymbol{\zeta} \mid \boldsymbol{\xi}]=\int \frac{1}{2}(\boldsymbol{\zeta}-\boldsymbol{\xi})^{\mathrm{T}} \boldsymbol{C}(\boldsymbol{\zeta}-\boldsymbol{\xi}) \mathrm{d}^{2} x+\frac{1}{(2 \pi)^{2}} \int \frac{1}{2} \hat{\boldsymbol{\zeta}}^{\mathrm{T}} \boldsymbol{A} \hat{\boldsymbol{\zeta}}^{*} \mathrm{~d}^{2} k
$$

The stable configurations of the dislocation ensemble may be identified with the relative minima of the energy (2.19), leading to the minimization problem

$$
\inf _{\zeta \in Y} \inf _{\xi \in X} E[\zeta \mid \xi]
$$

where $Y$ is some suitable configuration space for the slip fields. At this point we note that the energy (2.20) is quadratic in the multi-slip field $\zeta$. Therefore, the minimization with respect to this field can be carried out explicitly. To this end, we apply Parseval's identity to (2.20) to obtain

$E[\zeta \mid \xi]=\frac{1}{(2 \pi)^{2}} \int \frac{1}{2}(\hat{\boldsymbol{\zeta}}-\hat{\boldsymbol{\xi}})^{\mathrm{T}} \boldsymbol{C}\left(\hat{\boldsymbol{\zeta}}^{*}-\hat{\boldsymbol{\xi}}^{*}\right) \mathrm{d}^{2} k+\frac{1}{(2 \pi)^{2}} \int \frac{1}{2} \hat{\boldsymbol{\zeta}}^{\mathrm{T}} \boldsymbol{A} \hat{\boldsymbol{\zeta}}^{*} \mathrm{~d}^{2} k$.

The minimization with respect to $\hat{\zeta}$ can now be effected pointwise, with the result

$$
\hat{\zeta}=(\boldsymbol{A}+\boldsymbol{C})^{-1} \boldsymbol{C} \hat{\boldsymbol{\xi}}
$$

and

$$
E[\boldsymbol{\xi}]=\inf _{\boldsymbol{\xi} \in Y} E[\boldsymbol{\zeta} \mid \boldsymbol{\xi}]=\frac{1}{(2 \pi)^{2}} \int \frac{1}{2} \hat{\boldsymbol{\xi}}^{\mathrm{T}}\left[\boldsymbol{C}-\boldsymbol{C}(\boldsymbol{A}+\boldsymbol{C})^{-1} \boldsymbol{C}\right] \hat{\boldsymbol{\xi}}^{*} \mathrm{~d}^{2} k
$$

which expresses the total energy of the crystal in terms of the multi-phase field $\boldsymbol{\xi}$. The elastic interaction matrix $\boldsymbol{A}$ is homogeneous of degree 1 in $\boldsymbol{k}$, and hence $(\boldsymbol{A}+\boldsymbol{C})^{-1} \boldsymbol{C}=\boldsymbol{I}$ at $\boldsymbol{k}=\mathbf{0}$ and $(\boldsymbol{A}+\boldsymbol{C})^{-1} \boldsymbol{C} \rightarrow \mathbf{0}$ for $|\boldsymbol{k}| \rightarrow \infty$. Hence, the operator (2.23), which maps $\boldsymbol{\xi}$ to $\boldsymbol{\zeta}$, is a mollifier whose effect is to smooth out the core regions.

The problem of determining energy-minimizing dislocation networks within a slip plane is now reduced to minimizing the energy (2.24) with respect to the integer-valued multi-phase field $\xi$ under the action of applied forcing, e.g. in the form of an applied shear stress; in the presence of obstacles to the motion of dislocations, such as second-phase particles and secondary dislocations or under the action of a prescribed crystallographic misorientation across the plane, e.g. at a grain or sub-grain boundary. This latter case is considered in the remainder of this paper.

\section{Simulation of twist-boundary arrays}

In this section we apply the general framework developed in the foregoing to the simulation of a periodic array of finite twist boundaries. The displacement jump across an unrelaxed small-angle twist boundary coincident with the $\left(x_{1}, x_{2}\right)$-plane is

$$
\llbracket \boldsymbol{u} \rrbracket\left(x_{1}, x_{2}\right)=\theta\left(-x_{2}, x_{1}\right)=\theta \boldsymbol{e}_{3} \times \boldsymbol{x},
$$

where $\theta$ is the twist angle and $\boldsymbol{e}_{3}$ is the unit normal to the slip plane. This slip distribution must be matched on average by the displacement jump (2.10) resulting from the multi-phase 
field. This kinematic constraint drives the development of dislocation networks on the twist boundary.

The specific geometry under consideration consists of a periodic array of square grain boundaries of size $L$ on the $\left(x_{1}, x_{2}\right)$-plane, each of which is forced to undergo a prescribed twist angle $\theta$. In calculations, we enforce the constraint in the following average sense:

$$
\int_{B}\left(\sum_{\alpha=1}^{n} \xi^{\alpha} \boldsymbol{b}^{\alpha}-\theta \boldsymbol{e}_{3} \times \boldsymbol{x}\right) \mathrm{d}^{2} x=0,
$$

where $B$ is the prescribed twist region. The problem then is to minimize the energy (2.24) of the crystal with respect to the integer-valued multi-phase field $\boldsymbol{\xi}$ subject to the constraint (3.2). Equivalently, we may demand the stationarity of the Lagrangian

$$
L[\boldsymbol{\xi}, \boldsymbol{\tau}]=E[\boldsymbol{\xi}]-\boldsymbol{\tau} \cdot \int_{B}\left(\sum_{\alpha=1}^{n} \xi^{\alpha} \boldsymbol{b}^{\alpha}-\theta \boldsymbol{e}_{3} \times \boldsymbol{x}\right) \mathrm{d}^{2} x,
$$

where $\tau$ are Lagrange multipliers that may be interpreted as resolved shear stresses.

It is instructive to express the Lagrangian in dimensionless variables. To this end, suppose for simplicity that the Burgers vector length is the same for all systems. Introduce dimensionless coordinates $\tilde{x}_{i}=x_{i} / L$ and energy $\tilde{E}=E /\left(\mu b^{2} L^{2} / d\right)$, where $\mu$ is a representative shear modulus. Then the normalized Lagrangian takes the form

$$
\tilde{L}[\boldsymbol{\xi}, \tilde{\boldsymbol{\tau}}]=\tilde{E}[\boldsymbol{\xi}]-\tilde{\boldsymbol{\tau}} \cdot \int_{\tilde{B}}\left(\sum_{\alpha=1}^{n} \xi^{\alpha} \boldsymbol{s}^{\alpha}-\tilde{\theta} \boldsymbol{e}_{3} \times \tilde{\boldsymbol{x}}\right) \mathrm{d}^{2} \tilde{x},
$$

where $\tilde{\tau}=\tau /(\mu b / d)$ and $\tilde{\theta}=\theta /(b / L)$. This simple dimensional analysis shows that the solution depends on the twist angle, $\theta$, through the dimensionless group $\theta /(b / L)$, and consequently the effect of scaling the twist angle, $\theta$, at fixed $b$ and $L$ is identical to the effect of an equal scaling of $L$ or $b^{-1}$ at fixed $\theta$.

A cursory inspection of the normalized energy, $\tilde{E}[\xi]$, shows that it depends sensitively on the dimensionless parameter $L / d$. This dependence arises from the competition between the elastic and interplanar energies and illustrates the effect of confinement. Two welldifferentiated regimes may be identified in the behaviour of the system as a function of the parameter $L$. Thus for small $L / d$, the dislocation cores overlap and network formation is suppressed. This regime may be of relevance to nanostructured crystals. Conversely, for large $L / d$ it is possible to fit networks consisting of well-separated dislocation within the cell, and dislocation networks become a likely competitor for energy minimization. The constrained minimization problem (3.4) can be solved in closed form for infinite size domains. We also solve the problem for finite size twist boundaries using a Metropolis Monte Carlo algorithm [20-22]. A chief advantage of the present formulation is that it is amenable to the application of analytical tools such as the Fourier transform. This greatly facilitates the computation of the long-range elastic energy of the crystal, which is local in Fourier space. By contrast, the integer-valuedness constraint of the phase fields is most naturally enforced in real space, and in calculations we alternate between both representations through the use of the fast Fourier transform. The Metropolis Monte Carlo algorithm generates random multi-phase fields in accordance with the distribution $\exp \{-\beta L[\xi, \tau]\}$, where $\beta=1 / k_{\mathrm{B}} T$. We follow a standard simulated annealing approach, i.e. the calculations start at a high temperature, $T$, in order to thermalize the system, and subsequently the temperature is lowered in order to find low energy configurations.

For definiteness, we consider a cell size $[-L, L]=1000 \mathrm{~b}$. The prescribed twist region is chosen to be the circle of radius $r=L / 2$ in order not to have overlap between dislocations 
corresponding to neighbour cells. The phase fields are sampled on a grid of size $h=10 \mathrm{~b}$ and containing $10^{4}$ grid points. The range of the rotation angle in the simulations is limited by the size of the cell and grid. Following Frank's rule $[4,11,13]$, the distance between dislocations in a twist boundary is $D=b / \theta$; therefore we need the grid size to be smaller than $D$, and the cell size should satisfy $L / 2>D$. For simplicity, the crystal is assumed to be elastically isotropic. The values of the parameters are chosen to represent copper, i.e. $\mu=5.5 \times 10^{10} \mathrm{~N} \mathrm{~m}^{-2}$, $v=0.324$, and $b=2.56 \times 10^{-10} \mathrm{~m}$, where $\mu$ is the shear modulus and $v$ denotes Poisson's ratio.

\subsection{Square networks}

In keeping with observation [3-5], we consider square networks on (001)-planes resulting from activity on two systems defined by the pair of Burgers vectors

$$
\begin{aligned}
& \boldsymbol{b}_{1}=\frac{b}{2}(1,0,0), \\
& \boldsymbol{b}_{2}=\frac{b}{2}(0,1,0) .
\end{aligned}
$$

For this geometry, (2.10) specializes to

$$
\begin{aligned}
& \llbracket u_{1} \rrbracket=b \zeta^{1}, \\
& \llbracket u_{2} \rrbracket=b \zeta^{2},
\end{aligned}
$$

whereas the interaction matrix (2.12) evaluates to

$$
\boldsymbol{A}=\mu b^{2}\left(\begin{array}{cc}
\frac{k_{2}^{2}}{\sqrt{k_{1}^{2}+k_{2}^{2}}}+\frac{1}{1-v} \frac{k_{1}^{2}}{\sqrt{k_{1}^{2}+k_{2}^{2}}} & \frac{v}{2-2 v} \frac{k_{1} k_{2}}{\sqrt{k_{1}^{2}+k_{2}^{2}}} \\
\frac{v}{2-2 v} \frac{k_{1} k_{2}}{\sqrt{k_{1}^{2}+k_{2}^{2}}} & \frac{k_{1}^{2}}{\sqrt{k_{1}^{2}+k_{2}^{2}}}+\frac{1}{1-v} \frac{k_{2}^{2}}{\sqrt{k_{1}^{2}+k_{2}^{2}}}
\end{array}\right) .
$$

Figure 1 shows the computed low-energy dislocation configurations for twist angles $\theta=0.01$ and 0.05 . The dislocation ensemble consists of two species of dislocations belonging to each of the active slip systems. In the plot the dislocations are identified as the lines across which the corresponding phase field jumps by one. It is interesting to note that the dislocations form two main structures: a dislocation pile-up tracing the circle $r=L / 2$, which bounds the region where a twist is prescribed and a regular square network inside the twist region. The topology of the dislocation loops is clearly evident in figure 1(a) and, upon careful inspection, in figure $1(b)$ as well. Thus, the dislocation loops consist of arcs of circle closed off by the corresponding chords, bounding lenticular slip regions of constant phase field. The chords are aligned with the Burgers vectors and thus consist of screw segments. Two families of loops are superposed, corresponding to activity in each of the systems and to chords in each of the directions of the square network. A sequence of lenticular slip regions of varying depths are stacked so that the circular segments pile-up tightly and the corresponding chords form an evenly spaced square network.

By way of reference we note that an infinite twist boundary can be accommodated by means of a regular square network. The corresponding phase fields follow from (3.1), (3.6a), and (3.6b) as

$$
\begin{aligned}
& \xi^{1}=P_{\mathbb{Z}}\left(-\frac{\theta x_{2}}{b}\right), \\
& \xi^{2}=P_{\mathbb{Z}}\left(\frac{\theta x_{1}}{b}\right),
\end{aligned}
$$


(a)

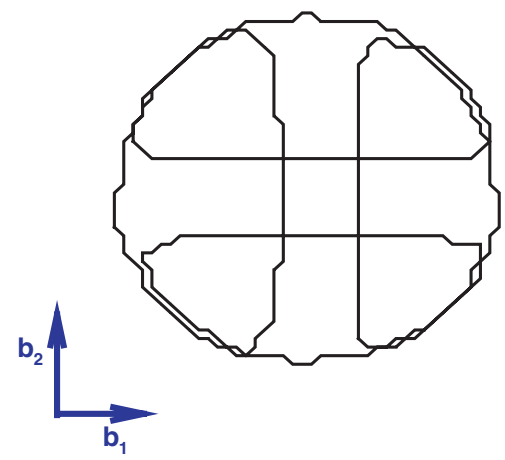

$\theta=0.01$

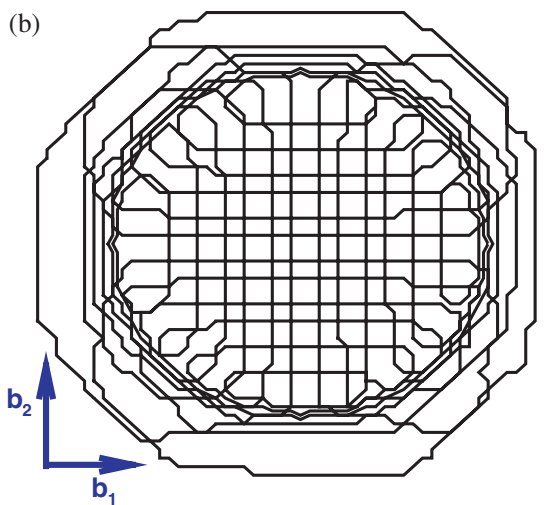

(b) $\theta=0.05$

Figure 1. Low-energy dislocation configuration in a periodic array of twist boundaries, consisting of a regular square network in the interior of the twist region and dislocation pile-up along its boundary.

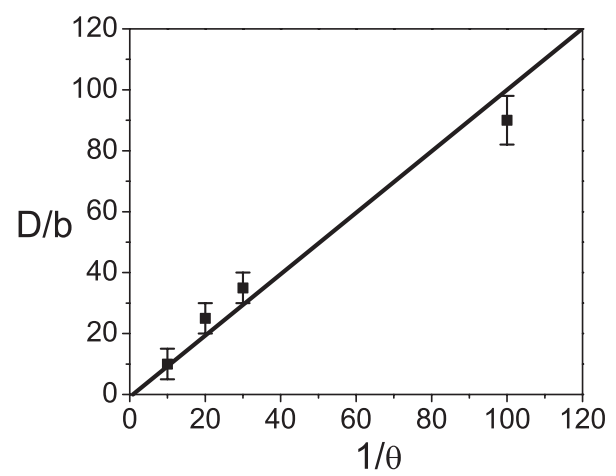

Figure 2. Dislocation spacing within the square dislocation networks forming in a periodic array of twist boundaries.

where $P_{\mathbb{Z}}$ denotes the closest-integer projection. These phase fields represent arrays of parallel dislocations at a distance

$$
D=\frac{b}{\theta}
$$

in agreement with Frank's relation (e.g. [11,13]). Figure 2 compares the dislocation separations computed for a twist-boundary array with those predicted by Frank's relation for an infinite twist boundary, equation (3.9). As is evident in the figure, within the parametric range covered by the calculations the computed dislocation separation follows Frank's relation closely.

The geometry and topology of the dislocation configuration resulting from the calculations can be given an energetic and geometrical interpretation. First, the topological constraint that dislocations form closed loops or networks is satisfied by the stacking of closed lenticular loops. The geometry of the loops serves two main purposes. First, the circular arc pile-up accommodates the transition from slip to no slip across the boundary of the twist region. Second, the chord segments accommodate the slip over the twist region roughly in accordance with (3.8a) and (3.8b). The orientation of the chords as screw segments also owes to energy minimization as for $v>0$ screw segments have a lower energy than do edge segments. 
(a)

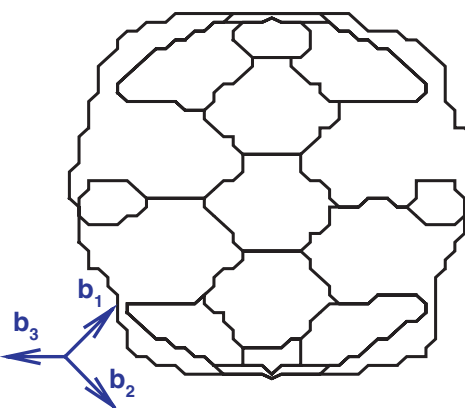

$\theta=0.01$ (b)

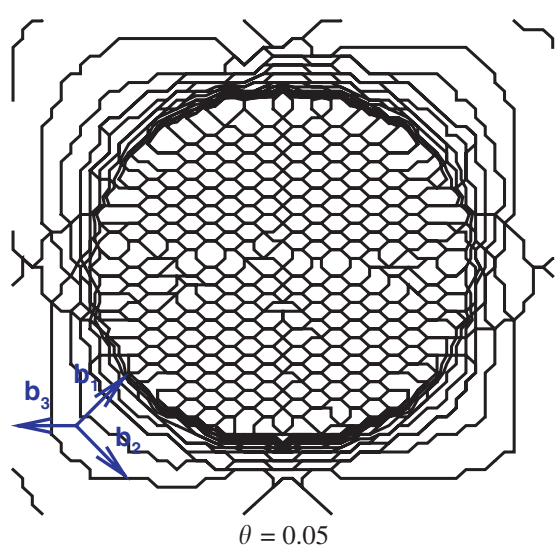

Figure 3. Low-energy dislocation configuration in a periodic array of twist boundaries, consisting of a regular hexagonal network in the interior of the twist region and dislocation pile-up along its boundary.

\subsection{Hexagonal networks}

According to theory and observation [3-5], in fcc crystals hexagonal networks are expected to form on (111)-planes owing to activity on three systems of Burgers vectors

$$
\begin{aligned}
& \boldsymbol{b}_{1}=b\left(\frac{1}{2}, \frac{\sqrt{3}}{2}, 0\right), \\
& \boldsymbol{b}_{2}=b\left(\frac{1}{2},-\frac{\sqrt{3}}{2}, 0\right), \\
& \boldsymbol{b}_{3}=\boldsymbol{b}_{1}+\boldsymbol{b}_{2},
\end{aligned}
$$

where all components refer to a reference frame in which the $x_{3}$-axis is normal to the plane of the network and the $x_{1}$ is aligned with $\boldsymbol{b}_{3}$. Evidently, only two of these vectors are linearly independent, and any displacement jump across the plane can be expressed as a linear combination of two Burgers vectors, e.g. $\boldsymbol{b}_{1}$ and $\boldsymbol{b}_{2}$. By virtue of this choice, (2.10) specializes to

$$
\begin{aligned}
& \llbracket u_{1} \rrbracket=\frac{1}{2} b \zeta^{1}+\frac{1}{2} b \zeta^{2}, \\
& \llbracket u_{2} \rrbracket=\frac{\sqrt{3}}{2} b \zeta^{1}-\frac{\sqrt{3}}{2} b \zeta^{2},
\end{aligned}
$$

whereas the interaction matrix (2.12) evaluates to

$$
\boldsymbol{A}=\mu b^{2}\left(\begin{array}{cc}
\frac{(4-3 v) k_{1}^{2}+(4-v) k_{2}^{2}-2 \sqrt{3} v k_{1} k_{2}}{8(1-v) \sqrt{k_{1}^{2}+k_{2}^{2}}} & \frac{(2-3 v) k_{1}^{2}+(2+v) k_{2}^{2}}{8(1-v) \sqrt{k_{1}^{2}+k_{2}^{2}}} \\
\frac{(2-3 v) k_{1}^{2}+(2+v) k_{2}^{2}}{8(1-v) \sqrt{k_{1}^{2}+k_{2}^{2}}} & \frac{(4-3 v) k_{1}^{2}+(4-v) k_{2}^{2}+2 \sqrt{3} v k_{1} k_{2}}{8(1-v) \sqrt{k_{1}^{2}+k_{2}^{2}}}
\end{array}\right) .
$$

Figure 3 shows the computed low-energy dislocation configurations for twist angles $\theta=0.01$ and 0.05 . The dislocation ensemble consists of three species of dislocations belonging 


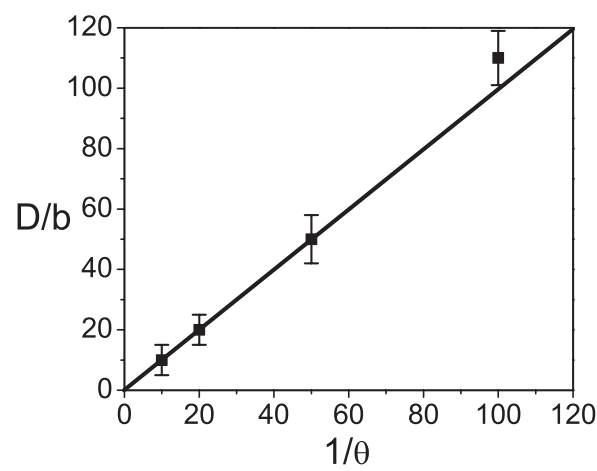

Figure 4. Dislocation spacing within the hexagonal dislocation networks forming in a periodic array of twist boundaries.

to each of the three active slip systems. In the plot the dislocations are identified as the lines across which the one of the phase fields jumps by one. Since the dislocations trace the level contours of a function, they necessarily form either networks or closed loops. Indeed, as in the case of the square networks, the dislocations form two main structures: a regular hexagonal network inside the twist region and a pile-up of dislocation arcs that close the end segments of the network. All the dislocation segments within the network have a screw character, as expected from energy minimization.

Again we note by way of reference that an infinite twist boundary can be accommodated by means of a regular hexagonal network. The corresponding phase fields follow from (3.1), $(3.11 a)$, and $(3.11 b)$ as

$$
\begin{aligned}
& \xi^{1}=P_{\mathbb{Z}}\left(\left(\frac{\theta}{b}\right)\left(\frac{x_{1}}{\sqrt{3}}-x_{2}\right)\right), \\
& \xi^{2}=P_{\mathbb{Z}}\left(\left(\frac{\theta}{b}\right)\left(-\frac{x_{1}}{\sqrt{3}}-x_{2}\right)\right),
\end{aligned}
$$

where, as before, $P_{\mathbb{Z}}$ denotes the closest-integer projection. These phase fields represent a hexagonal array of parallel dislocations at a distance

$$
D=\frac{b}{\theta}
$$

in agreement with Frank's relation. Figure 4 compares the dislocation separations computed for a twist-boundary array with those predicted by Frank's relation for an infinite twist boundary, equation (3.14). As is evident in the figure, within the parametric range covered by the calculations the computed dislocation separation follows Frank's relation closely.

\section{Summary and concluding remarks}

We have generalized the phase-field model of Koslowski et al [2] to processes requiring the activation of multiple slip systems on a slip plane. The energetics accounted for in the model include the core energy of the dislocations and the long-range elastic interaction between the dislocations in the slip plane. For infinite domains we find a closed form solution for square and hexagonal networks. The closest-integer projection renders the formation of networks that are in agreement with observations of twist boundaries [3,4] with the correct dislocation 
spacing. This is a clear advantage over approaches based on noninteracting dislocations or a line-tension approximation $[4,11,13]$.

The scope of the approach has also been demonstrated by means of an application concerned with the finite twist-boundary arrays. The theory predicts true dislocation networks in that the cores of dislocations of different types interact so as to form low-energy nodes. The simulated dislocation patterns are not affected by the grid size, $h$, given that the sampling grid is small enough to capture the dislocation spacing, i.e. $h \leqslant D$.

The dislocations in the patterns are of pure screw character, as expected from energetics, and the spacing between the dislocations in the network is in agreement with Frank's relation. In closing, we note that an advantage — as well as a limitation-of energy minimization as a governing principle is that it naturally predicts the equilibrium dislocation structures independent of initial conditions or distribution of sources.

\section{Acknowledgment}

We are grateful to the Department of Energy for support provided through Caltech's ASCI/ASAP Center for the Simulation of the Dynamic Response of Solids.

\section{References}

[1] Ortiz M 1999 Plastic yielding as a phase transition J. Appl. Mech. ASME 66 289-98

[2] Koslowski M, Cuitiño A and Ortiz M 2001 A phase-field theory of dislocations dynamics, strain hardening and hysteresis in ductile single crystals J. Mech. Phys. Solids 50 2597-635

[3] Amelinckx S 1956 The direct observation of dislocation patterns in transparent crystals Dislocation and Mechanical Properties of Crystals ed J C Fisher et al (New York: Wiley) pp 3-54

[4] Amelinckx S 1964 The direct observation of dislocations Solid State Physics vol 6, ed F Seitz and D Turnbull (New York: Academic)

[5] Sutton A P and Balluffi R W 1995 Interfaces in Crystalline Materials (Oxford: Oxford Science Publications)

[6] Leo P H and Hu J 1995 A continuum description of partially coherent interfaces Continuum Mech. Thermodyn. 7 39-56

[7] Hu J F and Leo P H 1997 Defect structures at thin film-substrate interfaces J. Mech. Phys. Solids 45 637-65

[8] Garroni A and Müller S $2003 \gamma$-limit of a phase-field model dislocations Preprint 92, Max Planck Institut für Mathematik in den Naturwissenschaften, Leipzig

[9] Bulatov V and Cai W 2002 Nodal effects in dislocation mobility Phys. Rev. Lett. 11115501

[10] Nabarro F R N 2001 Sequence of dislocation patterns Mater. Sci. Eng. A 317 12-16

[11] Hirth J P and Lothe J 1968 Theory of Dislocations (New York: McGraw-Hill)

[12] Kuhlmann-Wilsdorf D and van der Merve J H 1982 Mater. Sci. Eng. 5579

[13] Hull D and Bacon D J 1984 Introduction to dislocations International Series on Materials Science and Technology 3rd edn, vol 37 (Amsterdam: Elsevier)

[14] Gulluoglu A N, Srolovitz D J, LeSar R and Lomdahl P S 1989 Dislocation distributions in two dimensions Scr. Metall. 23 1347-52

[15] Hirth J P and Carnahan B 1991 Anisotropic elastic fields of twist boundaries Acta Metall. Mater. $401237-42$

[16] Argaman N, Levy O and Makov G 2001 Dislocation pattern formation-simulations of annealing in two dimensions J. Phys. IV 11 61-8

[17] Nye J F 1953 Some geometrical relations in dislocated crystals Acta Metall. 1 153-62

[18] Mura T 1987 Micromechanics of Defects in Solids (Boston: Kluwer)

[19] Ortiz M and Phillips R 1999 Nanomechanics of defects in solids Adv. Appl. Mech. 36 1-79

[20] Binder K (ed) 1986 Monte Carlo Methods in Statistical Physics 2nd edn (New York: Springer)

[21] Binder K (ed) 1992 The Monte Carlo Method in Condensed Matter Physics (New York: Springer)

[22] Thijssen J M 1999 Computational Physics (Cambridge: Cambridge University Press) 